\title{
The Study on the Segmentation of Remote Sensing Cloud Imagery
}

\author{
${ }^{1,3}$ Kai Liu*, ${ }^{2,3}$ Zheng Kou \\ 1 School of Computer Science \& Engineering, Southeast University, China \\ 2 National Key Laboratory on Electromagnetic Environmental Effects and Electro-optical \\ Engineering, PLA University of Science and Technology \\ 3 Institute of Meteorology and Oceanography, PLA University of Science and Technology, China
}

\begin{abstract}
The researches on weather system are very complicated because the weather system includes many factors, among which the cloud is a very important factor. Cloud appears with various shapes, different gray scales and not clear boundaries in the remote sensing images. For the active contour model and its extensions proposed by Vese and Chan can detect objects whose boundaries are not clear and not necessarily defined by gradient, we employ the active contour model in cloud segmentation in remote sensing cloud imagery. After the contours evolution, the image is automatically decomposed into a set of coherent regions with similar gray scale. The segmentation results are helpful to obtain the distribution of cloud at different levels as a whole, and moreover, the segmentation results could lay a good foundation for further image processing.
\end{abstract}

Keyword: image segmentation; remote sensing cloud image; active contour model without edges; multi-phase active contour model.

\section{Introduction}

The meteorological satellite imagery has been widely utilized in weather service with its high temporal and spatial resolution as well as extensive coverage up to date. It has become the most important unconventional data except for conventional data. Cloud is the vital target in meteorological satellite imagery. The reflec- 
tivity and emissivity of the clouds within the electromagnetic spectrum are ${ }^{1}$ $\mathrm{re}^{2}$ ferred to as the spectral properties of the clouds. These spectral properties are functions of cloud physical properties such as phase, thickness, and cloud top temperature [1]. Cloud top temperature is related to the height of the clouds, and higher clouds are cooler than lower clouds. Generally speaking, clouds are classified into three types, i.e. low level, middle level and high level. IR channels (IR10.8 and IR12.0) are useful for revealing differences in the temperature of cloud surfaces which are related to cloud top height [2]. Since the cloud classification is of vital importance for weather analysis and forecasting [3], the automatic cloud classification and segmentation have become a major research direction recently. In the past few years, several approaches of cloud classification, which based on different features and classifiers, have been developed and applied to images of various meteorological satellites such as AVHRR, MODIS, GMS etc. The early approaches used a set of thresholds on reflectance, brightness temperature (BT), and BT difference [4-7]. The subsequent approach of cloud classification is based on statistical and artificial neural network methods using features of radiance, reflectance, and BT [3], [8-12]. Recently, the singular value decomposition (SVD) is used to segregate cloud and non-cloud in visible and IR bands of imagery, and it can also classify clouds as low, medium or high level [13]. Furthermore, Ahmed AK. Tahir [2] proposes an approach for continuous daytime cloud classification system through satellite images. The system is based on spectral ratio values as input features and a modified version of probabilistic neural network (PNN), named Quick PNN (QPNN), as a classifier. The above mentioned methods based on statistical and artificial neural network involved two stages feature extraction and classification. These methods always could get higher classification accuracy when they are applied in a certain kind of satellite images, in case the satellite changed, the results would not be ideal, that is to say the universality of method is deficient. Moreover, the factors, such as noise in image, variable background intensity, and irregularity of cloud etc., all would affect the accuracy of classification, and the whole procedure was somewhat time exhausting and data dependent. Nevertheless, a type of image segmentation approaches based on active contour model has been applied in medical image, remote sensing image and industrial image. The wide applicability of active contours is attributed to the inherent continuity and smoothness of active contours. The model can compensate for noise, gaps and irregularities in object boundaries. In this paper, we employ the multi-phase active contour model for segmentation in satellite cloud imagery, and obtain a set of cloud segments of different levels hoping to offer some help to weather analysis for forecasters.

\footnotetext{
${ }^{1}$ Kai. Liu ( $\left.\square\right)$

School of Computer Science \& Engineering, Southeast University, Nanjing, , China e-mail: liukaiqy@163.com
} 
The paper is organized as follows. In section 1, we introduce the multi-phase active contour model and related model. In section 2, we apply the 4-phases level set active contour model for cloud segmentation in IR band images and present the results of tests. And conclusion is given in section 4 .

\section{Multi-phase active contours model and related model}

\subsection{Active contour model without edges}

Chan and Vese [14] proposed the active contour model without edges, which is also called C-V model, and it is a particular case of the Mumford and Shah model. Given the curve $C=\partial \omega$, with $\omega \subset \Omega$ an open subset, and two unknown constants $c_{1}$ and $c_{2}$, denoting $\Omega_{1}=\omega, \Omega_{2}=\Omega \backslash \omega$, the energy functional $F\left(c_{1}, c_{2}, C\right)$ is defined by

$$
\begin{aligned}
& F\left(c_{1}, c_{2}, C\right)=\mu \cdot \text { Length }(C)+\lambda_{1} \int_{\text {inside }(C)}\left|u_{0}(x, y)-c_{1}\right|^{2} d x d y+ \\
& \lambda_{2} \int_{\text {outside }(C)}\left|u_{0}(x, y)-c_{2}\right|^{2} d x d y
\end{aligned}
$$

where $\mu \geq 0, \lambda_{1}, \lambda_{2}>0$ are fixed parameters.

The energy functional $F\left(c_{1}, c_{2}, C\right)$ is transformed in the level set formulation, with $C=\{(x, y) \mid \phi(x, y)=0\}$ :

$$
\begin{aligned}
& F\left(c_{1}, c_{2}, \phi\right)=\mu \int_{\Omega} \delta(\phi(x, y))|\nabla \phi(x, y)| d x d y+\lambda_{1} \int_{\Omega}\left|u_{0}(x, y)-c_{1}\right|^{2} H(\phi(x, y)) d x d y+ \\
& \lambda_{2} \int_{\Omega}\left|u_{0}(x, y)-c_{2}\right|^{2}(1-H(\phi(x, y))) d x d y
\end{aligned}
$$

keeps $\phi$ fixed and minimizes the energy functional with respect to the constants $C_{1}$ and $c_{2}$

$c_{1}(\phi)=\frac{\int_{\Omega} u_{0}(x, y) H(\phi(x, y)) d x d y}{\int_{\Omega} H(\phi(x, y)) d x d y}$ 
$c_{2}(\phi)=\frac{\int_{\Omega} u_{0}(x, y)(1-H(\phi(x, y))) d x d y}{\int_{\Omega}(1-H(\phi(x, y))) d x d y}$

keeps $c_{1}$ and $c_{2}$ fixed and minimizes the energy functional with respect to $\phi$, the associated Euler-Lagrange equation for $\phi$ was deduced:

$$
\frac{\partial \phi}{\partial t}=\delta_{\varepsilon}(\phi)\left[\mu d v\left(\frac{{ }^{\nabla} \phi}{|\nabla \phi|}\right)-\left(u_{0}-c_{1}\right)^{2}+\left(u_{0}-c_{2}\right)^{2}\right]
$$

\subsection{Multi-phase level set based active contour model}

The aforementioned C-V model uses only one level set function, so it can represent only two phases or segments in the image. For some other geometrical features, such as triple junctions and complex topologies, cannot be represented using only one level set function, Vese and Chan [15] endeavored to look for a new multi-phase level set model which can represent more than two segments or phases in an efficient way. They proposed using only $\log _{2} n$ level set functions to represent $n$ phases or segments.

They considered $m=\log _{2} n$ level set functions $\phi_{i}: \Omega \rightarrow R$. The union of the zero-level sets of $\phi_{i}$ represented the edges in the segmented image. They also introduced the "vector level set function" $\Phi=\left(\varphi_{1}, \ldots, \varphi_{\mathrm{m}}\right)$, and the "vector Heaviside function" $H(\Phi)=\left(H\left(\varphi_{1}\right), \ldots, H\left(\varphi_{\mathrm{m}}\right)\right)$. They labeled the classes by $I$, with $1 \leq I \leq 2^{m}=$ $n$ and defined a constant vector of averages $c=\left(c_{1}, \ldots, c_{\mathrm{n}}\right)$, where $C_{I}=\operatorname{mean}\left(u_{0}\right)$ in the class $I$, and the characteristic function $\chi_{I}$ for each class $I$. The energy function would be minimized was given by:

$$
F_{n}(c, \Phi)=\sum_{1 \leq I \leq n=2^{m}} \int_{\Omega}\left(u_{0}-c_{I}\right)^{2} \chi_{I} d x d y+\sum_{1 \leq I \leq m} \mu \int_{\Omega}\left|\nabla H\left(\phi_{i}\right)\right|
$$

For $n=2$, therefore $m=1$, the active contour without edges was obtained. For $n=4$ phases or classes, therefore $m=2$ level set functions:

$$
\begin{aligned}
& F_{4}(c, \Phi)=\int_{\Omega}\left(u_{0}-c_{11}\right)^{2} H\left(\phi_{1}\right) H\left(\phi_{2}\right) d x d y+\int_{\Omega}\left(u_{0}-c_{10}\right)^{2} H\left(\phi_{1}\right)\left(1-H\left(\phi_{2}\right)\right) d x d y \\
& +\int_{\Omega}\left(u_{0}-c_{01}\right)^{2}\left(1-H\left(\phi_{1}\right)\right) H\left(\phi_{2}\right) d x d y+\int_{\Omega}\left(u_{0}-c_{00}\right)^{2}\left(1-H\left(\phi_{1}\right)\right)\left(1-H\left(\phi_{2}\right)\right) d x d y \\
& +\mu \int_{\Omega}\left|\nabla H\left(\phi_{1}\right)\right|+\mu \int_{\Omega}\left|\nabla H\left(\phi_{2}\right)\right|
\end{aligned}
$$

where $c=\left(c_{11}, c_{10}, c_{01}, c_{00}\right)$ was constant vector, and $\Phi=\left(\Phi_{1}, \Phi_{2}\right)$. 


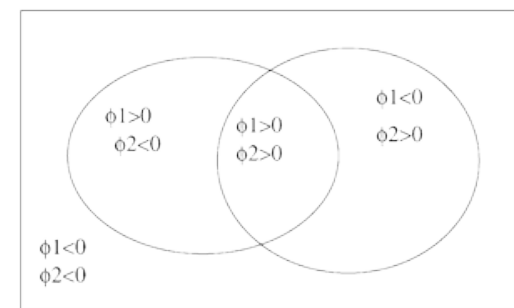

$c_{11}(\Phi)=\operatorname{mean}\left(u_{0}\right)$ in $\left\{(x, y): \Phi_{1}(t, x, y)>0, \Phi_{2}(t, x, y)>0\right\}$

$c_{10}(\Phi)=\operatorname{mean}\left(u_{0}\right)$ in $\left\{(x, y): \Phi_{1}(t, x, y)>0, \Phi_{2}(t, x, y)<0\right\}$

$c_{01}(\Phi)=\operatorname{mean}\left(u_{0}\right)$ in $\left\{(x, y): \Phi_{1}(t, x, y)<0, \Phi_{2}(t, x, y)>0\right\}$

$c_{10}(\Phi)=\operatorname{mean}\left(u_{0}\right)$ in $\left\{(x, y): \Phi_{1}(t, x, y)<0, \Phi_{2}(t, x, y)<0\right\}$

The Euler-Lagrange equations obtained by minimizing (7) with respect to $C$ and $\Phi:$

$\frac{\partial \phi_{1}}{\partial t}=\delta_{\varepsilon}\left(\phi_{1}\right)\left\{\mu d v\left(\frac{\nabla}{\mid \nabla \phi_{1}}\right)-\left[\left(\left(u_{0}-c_{11}\right)^{2}-\left(u_{0}-c_{01}\right)^{2}\right) H\left(\phi_{2}\right)\right.\right.$

$\left.\left.+\left(\left(u_{0}-c_{10}\right)^{2}-\left(u_{0}-c_{00}\right)^{2}\right)\left(1-H\left(\phi_{2}\right)\right)\right]\right\}$

$\frac{\partial \phi_{2}}{\partial t}=\delta_{\varepsilon}\left(\phi_{2}\right)\left\{\mu d v\left(\frac{\nabla \phi_{2}}{\left|\nabla \phi_{2}\right|}\right)-\left[\left(\left(u_{0}-c_{11}\right)^{2}-\left(u_{0}-c_{10}\right)^{2}\right) H\left(\phi_{1}\right)\right.\right.$

$\left.\left.+\left(\left(u_{0}-c_{01}\right)^{2}-\left(u_{0}-c_{00}\right)^{2}\right)\left(1-H\left(\phi_{1}\right)\right)\right]\right\}$

\section{Segmentation of satellite cloud imagery applying multi-phase active contours model}

The FY series geostationary meteorological satellites have five spectral bands: one visible band, one water vapor band, one near infrared band, two thermal infrared bands. The chosen test images are the china continental cloud images of IR1 band which downloaded from national satellite meteorological center (NSMC). The size of image is $256 \times 256$. We apply the multi-phase active contours model in the segmentation of two test images, and obtain four segments in the images. The main parameters in the model needed to be set are iteration number $(\mathrm{N}), \mu$ and the mask for initial contours.

Fig.1(a) and (b) are two IR1 band images, Fig.2(a) and (b) are two contours' transformation after a number of iterations, Fig.3(a) and (b) are two global regionbased segmentation of two images. 


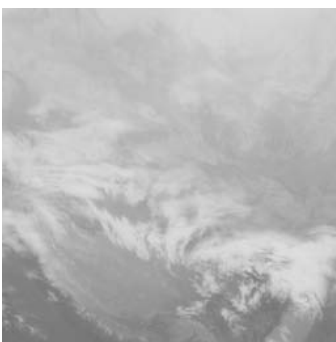

(a)

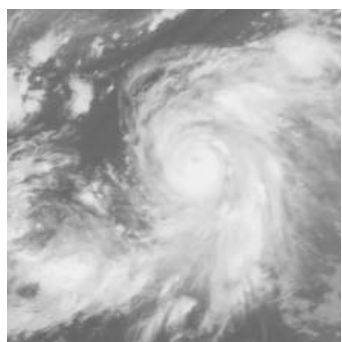

(b)

Fig.1: two IR1 band images

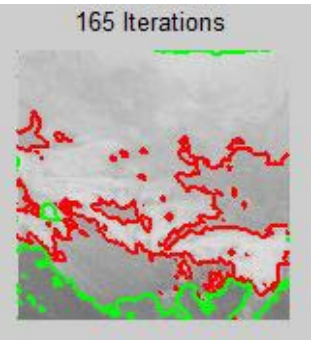

(a)

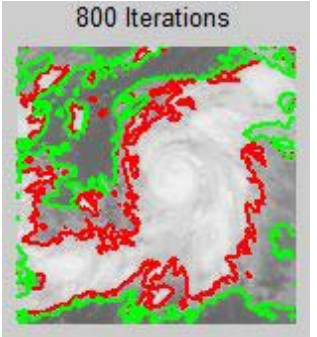

(b)

Fig.2: contours' transformation after iterations

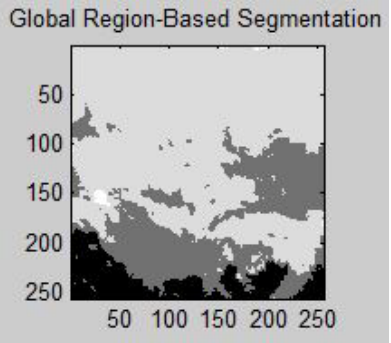

(a)

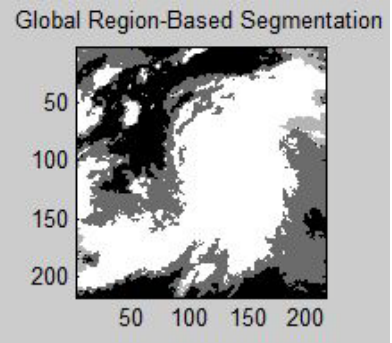

(b)

Fig.3: global region-based segmentation

As seen from Fig.3(a) and Fig.3(b), the images are segmented into four classes, each class is identified by the mean gray scale in closed region. Each of the four classes corresponds to cloud at different height levels, the higher the gray scale the higher the cloud top height, for the gray scale in IR channel image reveals the differences in temperature of cloud surfaces which are related to cloud top height. As shown in Fig.3(a) most of clouds are high level, the low level cloud and land surface or sea surface are in the second place, and middle level cloud is the less. As shown in Fig.3(b), the main body of typhoon cloud belt with very high gray level is well segmented from other cloud, after the further processing of the typhoon re- 
gion, we can locate the typhoon. So from the global region-based segmentation results we could understand the rough distribution of clouds at different levels in the image, and realize which type of clouds is dominant in the image, that would be helpful for the analysis of weather system.

\section{Conclusion}

In this paper we use the level set based multi-phase active contours model in segmentation of satellite cloud image. The model could automatically divide the image into four classes through the transformation and evolution of contours. The obtained the global region-based segmentation results facilitate understanding the distribution of cloud at different levels and offer some help for weather system analysis.

\section{Acknowledgements}

Thanks are due to Yue Wu of Tufts University for his valuable Matlab code file. Thanks are also due to the referees for reviewing the paper and giving valuable comments.

\section{Reference}

[1] I.Mamoru, Analysis and Use of Meteorological Satellite Images, 1st ed. Tokyo, Japan, Meteorol. Satell. Centre, Japan Meteorol. Agency, 2002.

[2] A.AK. Tahir, A System Based on Ratio Images and Quick Probabilistic Neural Network for ontinuous Cloud Classification, IEEE Trans. Geosci. Remote Sens.,

[3] K. Saitwal, M. R. Azemi-Sadjadi, D. Reinke, A multichannel temporally adaptive system for continuous cloud classification from satellite imagery, IEEE Trans. Geosci. Remote Sens., 41(5), 1098- 1104 (2003).

[4] Y. Fan, L. Changsheng, and C. Weimin, Man-computer interactive method on cloud classification based on bispectral satellite imagery, Adv. Atmos. Sci., 14(3), 389-398 (1997).

[5] S. A. Ackerman, K. I. Strabala, W. P. Menzel, R. A. Frey, C. C. Moeller, and L. E. Gumley, Discriminating clear sky from clouds with MODIS, J. Geophys. Res., 103(D24), 32 141-32 157(1998).

[6] B. Huang, S. A. Ackerman, W. P. Menzel, A new method forMODIS cloud classification, in Proc. SPIE, 5890, 210-216 (2005).

[7] P. Minnis, Q.Z. Trepte, S. Sun-Mack, C. Yan, et al., Cloud detection in nonpolar regions for CERES using TRMM VIRS and terra and aqua MODIS data, IEEE Trans. Geosci. Remote Sens., 46(11), 3857-3884 (2008).

[8] Y. Fan and A. Hao, Cloud classification by using multi-spectral GMS imagery and comparison with surface cloud observation, in Proc. SPIE, 7149,71490S-7-1490S-9 (2008). 
[9] J. Li, W. P. Menzel, Z. Yang, R. A. Frey, S. A. Ackerman, Highspatial- resolution surface and cloud-type classification fromMODIS multispectral band measurements, J. Appl. Meteorol., 42, 204-226 (2003).

[10] Z. Li, J. Li, W. P. Menzel, T. J. Schmit, S. A. Ackerman, Comparison between current and future environmental satellite imagers on cloud classification using MODIS, Remote Sens. Environ., 108(3), 311-326 (2007).

[11] L. Gomez-Chova, G. Camps-Valls, J. Calpe-Maravilla, L. Guanter, et al., Cloud-screening algorithm for ENVISAT/MERIS multispectral images, IEEE Trans. Geosci. Remote Sens., 45(12), 4105- 4118 (2007).

[12] L. Gomez-Chova, J. Muoz-Mari, E. Izquierdo-Verdiguier, G. Camps-Vails, J. Calpe, and J. Moreno, "Cloud screening with

combined MERIS and AATSR images," in Proc. IEEE IGARSS, Cape Town, South Africa, Jul. 12-17, 2009, pp. IV-761-IV-764.

[13] R. Kaur, A. Ganju, Cloud Classifi cation in NOAA AVHRR Imageries using Spectral and textural Features, J. Indian Soc. Remote Sens. , 36, 167-174 (2008).

[14] T.F. Chan, L.A. Vese, Active contour without edges, IEEE Transactions on Imaging Processing, 10(2), 266-277 (2001)

[15] L.A. Vese, T.F. Chan, A multi-phase level set frame work for image segmentation using the Mumford and Shah model , International Journal of Computer Vision, 50(3), 271-293 (2002). 